\title{
Validation of TIRADS in Evaluation of Thyroid Nodule
}

\author{
Medhat M. Reffat, Maha Z. Bilal, Mona N. Elbeshbishi, Mai M. Elrakhawy
}

\begin{abstract}
:
Department of radiology, Benha faculty of medicine, Banha University, Egypt.

Background: This study was performed to prospectively investigate the diagnostic reliability of the daily use of TIRADS classification Correspondence to: Mai M. Elrakhawy, Department of radiology, Benha faculty of medicine, Banha University, Egypt

Email: system, in differentiating between a benign and a malignant lesion. Patients and Methods: In this prospective observational study, 30 patients with thyroid nodules underwent ultrasound examination and fine needle aspiration. The ultrasound studies were evaluated maielrakhawy@yahoo.com Received: 27 April 2020

Accepted: 18 May 2021 according to the TIRADS characteristics of composition, echogenicity, margins, shape, and echogenic foci. Each feature in a particular USG characteristic was scored and ACR-TIRADS categorization done from 1 to 5 . This was compared to FNAC. Results: Of the 30 nodules included in the study, 23 were found to be benign and 7 were found to be malignant. Risk of malignancy for all ultrasound features showed an increasing trend with higher scored feature. All nodules in TIRADS 1,2 and 3 scores were benign, 1 out of 7 nodules in TIRADS 4 was malignant, all nodules in TIRADS 5 revealed malignancy. Colloid nodular goiter represented the most common benign pathology, while papillary thyroid carcinoma was the most common malignant entity. Conclusions: TIRADS is a high specific, accurate classification system for categorizing the thyroid nodules based on ultrasound features, for assessing the risk of malignancy.
\end{abstract}

Keywords: TIRADS, Ultrasound, Thyroid nodule.

List of abbreviations:

ACR American College of Radiology

TIRADS Thyroid Imaging Reporting and Data System

FNA Fine Needle Aspiration

PEF Punctate Echogenic Foci

PPV Positive Predictive Value 


\section{Introduction:}

Thyroid nodules are highly prevalent in the general population; most of them are asymptomatic and can only be identified by imaging techniques (1).

The clinical significance of thyroid nodules is related to the need to exclude thyroid cancer, which is found in $5-15 \%$ of cases, depending on sex, age, and exposure to other risk factors. The incidence of thyroid cancer has increased about fivefold in the last 50 years, mostly due to small papillary thyroid cancers, the most indolent form of thyroid cancer (2).

Ultrasonography (US) is widely used in the assessment of the thyroid gland. Among the different pathologies that can be depicted and characterized by US are nodules. Nodules can be benign or malignant (3).

Some US-features are in favor of benignity or malignity, especially when grouped together. Suspicious nodules will require fine needle aspiration biopsy (FNAB) for pathology analysis. Although some guidelines have been proposed, some confusion still exists as the same nodule may be classified differently using different guidelines implying different diagnostic or therapeutic attitudes (4).
Recently, the American College of Radiology (ACR) proposed a Thyroid Imaging Reporting and Data System (TIRADS) for thyroid nodules based on Ultrasonographic features that consist of five levels.in ACR TI-RADS, points in five feature categories are summed to determine a risk level from TR1 to TR5. Recommendations for biopsy or US followup are based on the nodule's ACR TI-RADS level and its maximum diameter (5).

\section{Aim of the work:}

The aim of the study is to assess the reliability of TIRADS in evaluation of thyroid nodule and its role in predicting thyroid malignancy

\section{Patients and methods:}

In a prospective study from March 2019 to January 2020 series of 30 patients (24 female and 6 male; age between $21-70$ years) underwent thyroid ultrasound as well as ultrasound-guided FNA for thyroid nodules at Benha University.

Ultrasound scan of the thyroid glands and neck areas was done by using a $7.5 \mathrm{MHz}$ linear array transducer (Toshiba Xario system). Ultrasound examination was 
performed with the patient in a supine position, with the neck slightly overextended by a pillow under the shoulders.

The scanning protocol in all cases included both transverse and longitudinal real time imaging of the thyroid gland and lymph nodes and color coded Doppler imaging.

We obtained informed written consent from all the patients before performing ultrasound-guided FNA. This study was approved by the institutional review board of our hospital.

\section{Image analysis}

Analysis of ultrasound and power Doppler images was performed. We were blinded to the other clinical and final pathological examinations of thyroid nodules. All thyroid nodules were characterized according to the internal component (solid, mixed or cystic), calcifications and the shape.
Margins were classified as well circumscribed, lobulated or irregular. Echogenicity was classified as "hyperechogenicity", "isoechogenicity", "hypoechogenicity" and "marked hypoechogenicity".

Isoechogenicity was defined as an echogenicity similar to that of the adjacent healthy thyroid gland. A nodule was classified as "marked hypoechogenicity" if the echogenicity was less than that of the superficial surrounding neck muscles. When present, calcifications were categorized as microcalcifications and macrocalcifications. The shape of the nodule was categorized as "taller than wide" (greater in its anteroposterior dimension than in its transverse dimension) and "wider than tall". 


\section{Results:}

Table (1) illustrate that $76.6 \%$ of the studied thyroid lesions were isoechoic and $13.3 \%$ were hypoechoic. In addition, $90 \%$ of the lesions were wider orientation and $70 \%$ had well defined borders. Echogenic foci distribution was as follow (53.3\% have no foci, 16.7 for punctate \& macro-calcification each and $13.3 \%$ comet tail).

\begin{tabular}{lll}
\hline & $\mathbf{N}=\mathbf{3 0}$ & \% \\
\hline Echogenicity & 2 & \\
$\begin{array}{l}\text { Anechoic } \\
\text { Hypoechoic }\end{array}$ & 4 & 6.7 \\
$\begin{array}{l}\text { Isoechoic } \\
\text { Hyper echoic }\end{array}$ & 23 & 13.3 \\
Orientation & 1 & 76.7 \\
Wider & & 3.3 \\
Taller & 27 & \\
Borders & 3 & 90.0 \\
Extra-thyroid & & 10.0 \\
Lobulated & 1 & 3.3 \\
Well defined & 4 & 13.3 \\
Irregular & 21 & 70.0 \\
& 4 & 13.3 \\
Echogenic foci & & \\
None & & 53.3 \\
Punctate echogenic foci & 16 & 16.7 \\
Macro-calcification & 5 & 16.7 \\
Comet tail & 5 & 13.3 \\
\hline
\end{tabular}

Table (2) shows that the most common pathological type of the studied lesions was colloid nodular goiter (46.7\%) followed by papillary thyroid carcinoma and colloid cyst ( $16.7 \%$ each) with the least commonly found lesion was medullary thyroid carcinoma $(3.3 \%)$

\begin{tabular}{lcc}
\hline \multicolumn{1}{c}{ Pathological types } & N=30 & \% \\
\hline Medullary thyroid carcinoma & 1 & 3.3 \\
Papillary thyroid carcinoma & 5 & 16.7 \\
Goiterous nodule nodule & 1 & 3.3 \\
Follicular carcinoma & 2 & 6.7 \\
Follicular adenoma & 2 & 6.7 \\
Colloid nodule goiter & 14 & 46.7 \\
Colloid cyst & 5 & 16.7 \\
\hline
\end{tabular}

Table (3) shows that $76.7 \%$ were benign lesions and $23.3 \%$ were malignant.

\begin{tabular}{lcc}
\hline Pathology & $\mathbf{N}=\mathbf{3 0}$ & \% \\
\hline Benign & 23 & 76.7 \\
Malignant & 7 & 23.3 \\
\hline
\end{tabular}


Benha medical journal, Vol. 38, issue 2, 2021

Table (4) demonstrates that there is statistically significant difference between benign and malignant lesion regarding TIRADS classification, with benign lesion was detected from TIRADS 1 to 4 ( $34.8 \%$ were TIRADS $2 \& 3$ , 26.1\% was TIRADS $26.1 \%$ and $.3 \%$ were TIRADS 1). Malignant lesions were distributed among TIRADS $4 \& 5$ (85.7\% were TIRADS 5and $14.3 \%$ ere TIRADS 4 ).

\begin{tabular}{|c|c|c|c|}
\hline \multirow[t]{2}{*}{ TIRADS } & \multicolumn{2}{|c|}{ Pathology } & \multirow[t]{2}{*}{ Test of significance } \\
\hline & $\begin{array}{c}\text { Benign } \\
\mathrm{N}=23(\%)\end{array}$ & $\begin{array}{c}\text { Malignant } \\
\mathrm{N}=7(\%)\end{array}$ & \\
\hline 1 & $1(4.3)$ & $0(0.0)$ & \\
\hline 2 & $8(34.8)$ & $0(0.0)$ & $\begin{array}{c}\mathrm{MC} \\
\mathrm{P}<0.001 *\end{array}$ \\
\hline 3 & $8(34.8)$ & $0(0.0)$ & \\
\hline 4 & $6(26.1)$ & $1(14.3)$ & \\
\hline 5 & $0(0.0)$ & $6(85.7)$ & \\
\hline
\end{tabular}

\section{Cases}

\section{Case (1)}

Female patient aged 34 years with left thyroid swelling.

\section{Ultrasound features of the nodule:}

A well-defined anechoic cystic nodule occupying most of left thyroid lobe with smooth margins. No detected calcifications. It measures about 16 × 20 × 25 mm.

\section{TIRADS level: TIRADS (1).}

\section{Pathology: Colloid cyst.}
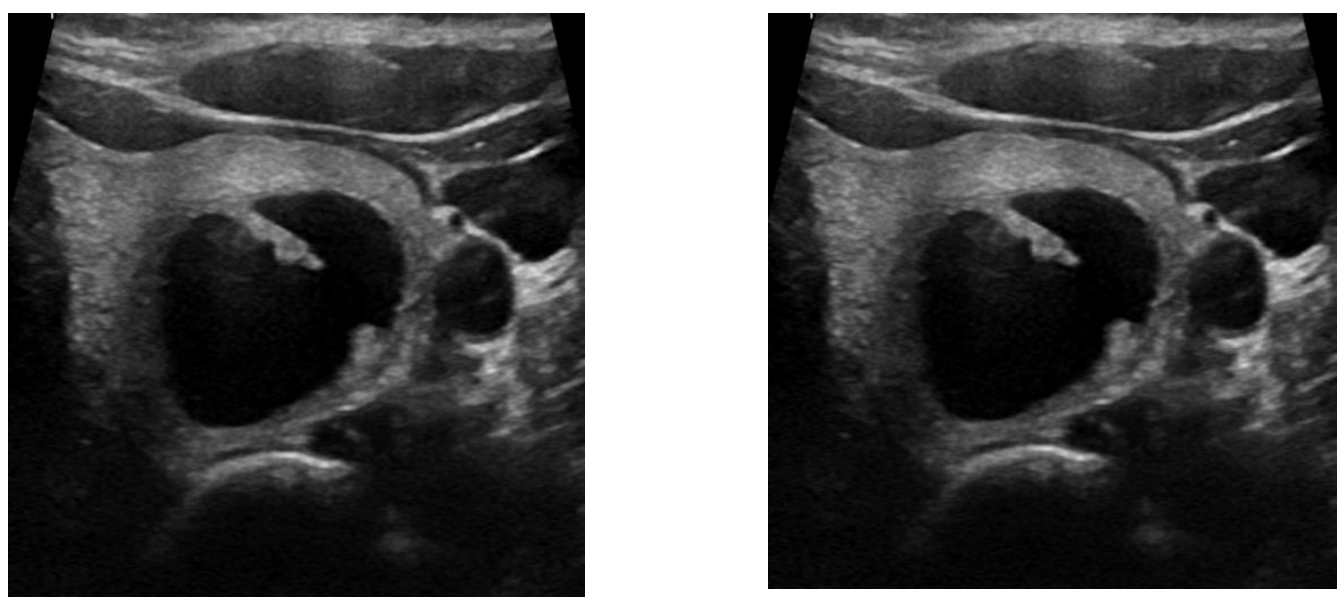


\section{Case (2)}

Female patient aged 51 years with right thyroid swelling.

\section{Ultrasound features of the nodule:}

- A well-defined partially cystic partially solid nodule occupying most of right thyroid lobe with smooth margins. Large comet tail artifact is seen at its cystic part. It measures about $16 \times 20 \times 21 \mathrm{~mm}$.

\section{TIRADS level: TIRADS (2).}

Pathology: Goitrous nodule.
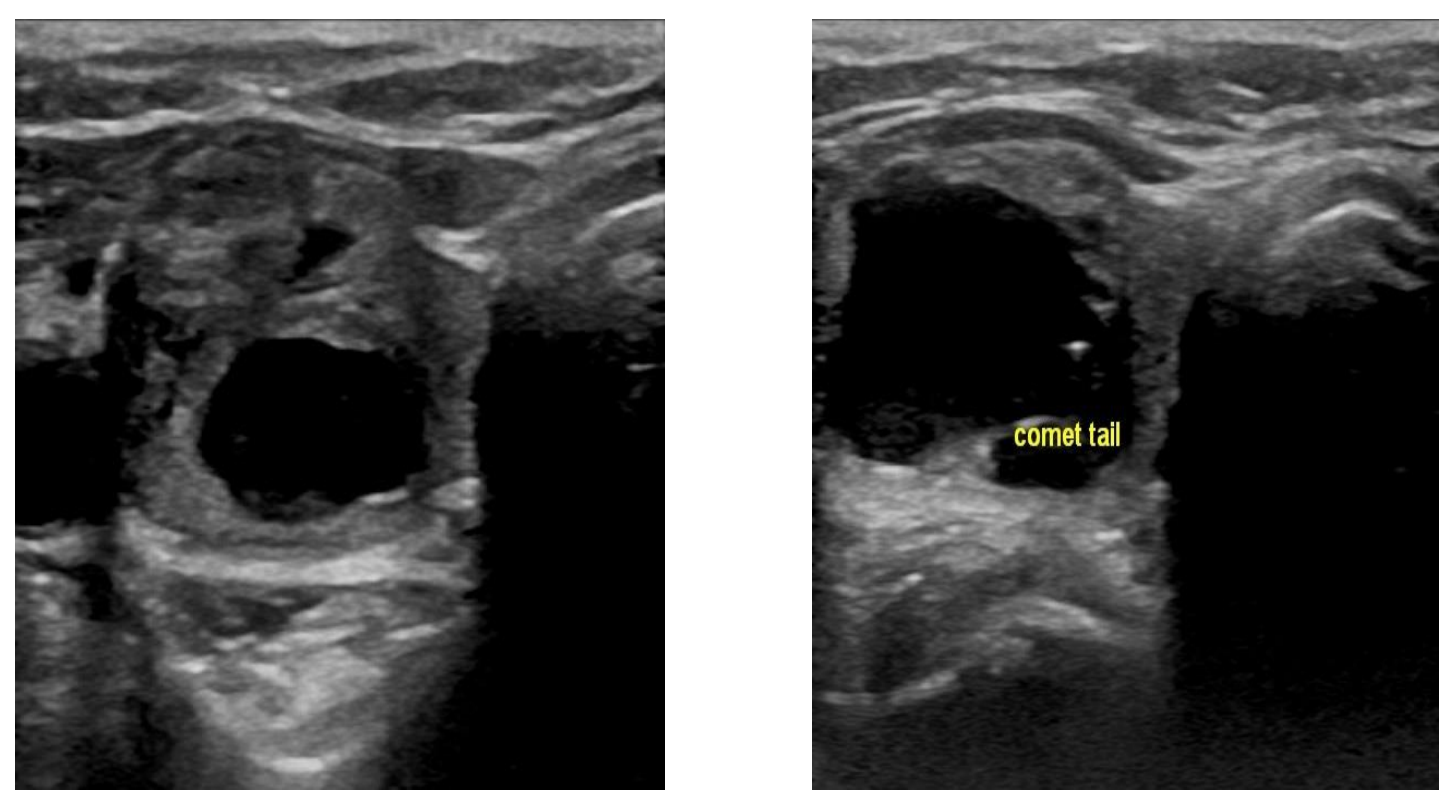

\section{Case (3)}

Female patient aged 68 years with left thyroid swelling.

\section{Ultrasound features of the nodule:}

- A well-defined solid isoechoic nodule is seen at left thyroid lobe with smooth margins. No detected echogenic foci. It measures about $11 \times 18 \times 26 \mathrm{~mm}$.

TIRADS level: TIRADS (3).

Pathology: Follicular adenoma. 

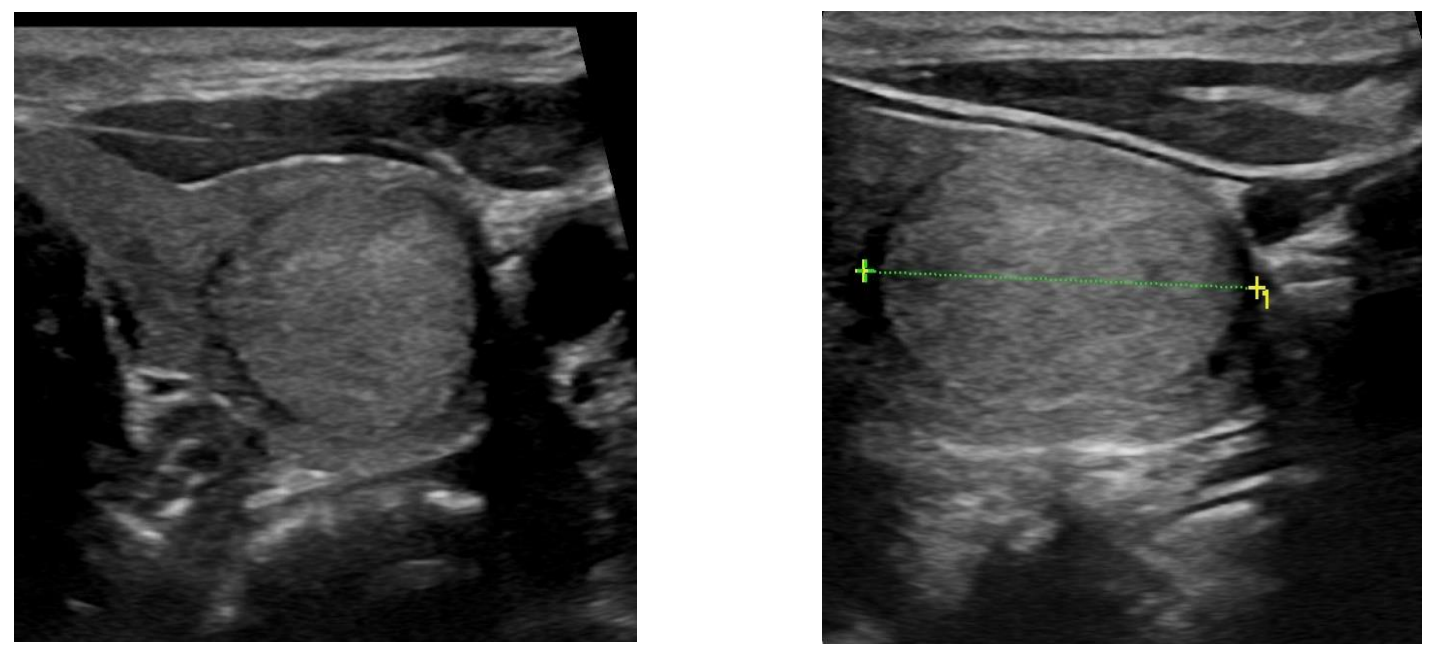

Case (4)

Female patient aged 53 years with right thyroid swelling.

\section{Ultrasound features of the nodule:}

- A well-defined solid isoechoic nodule with lobulated margins is seen occupying most of the right thyroid lobe. It measures about $24 \times 18 \times 30 \mathrm{~mm}$.

TIRADS level: TIRADS (4).

Pathology: Follicular thyroid carcinoma.
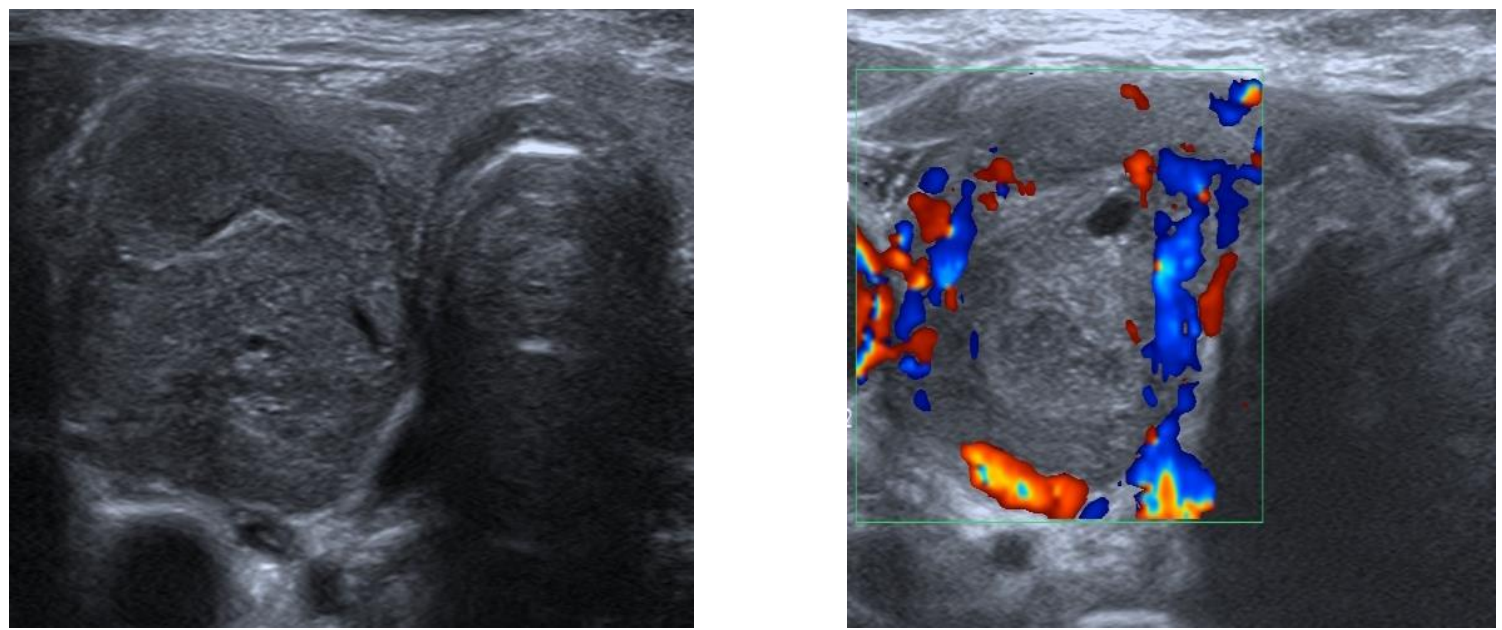


\section{Case (5)}

Male patient aged 40 years with right thyroid swelling.

\section{Ultrasound features of the nodule:}

- An ill-defined solid hypoechoic nodule with irregular margins and punctate echogenic foci is seen at right thyroid lobe. It measures about $11 \times 14 \times 25 \mathrm{~mm}$.

TIRADS level: TIRADS (5).

Pathology: Papillary thyroid carcinoma.
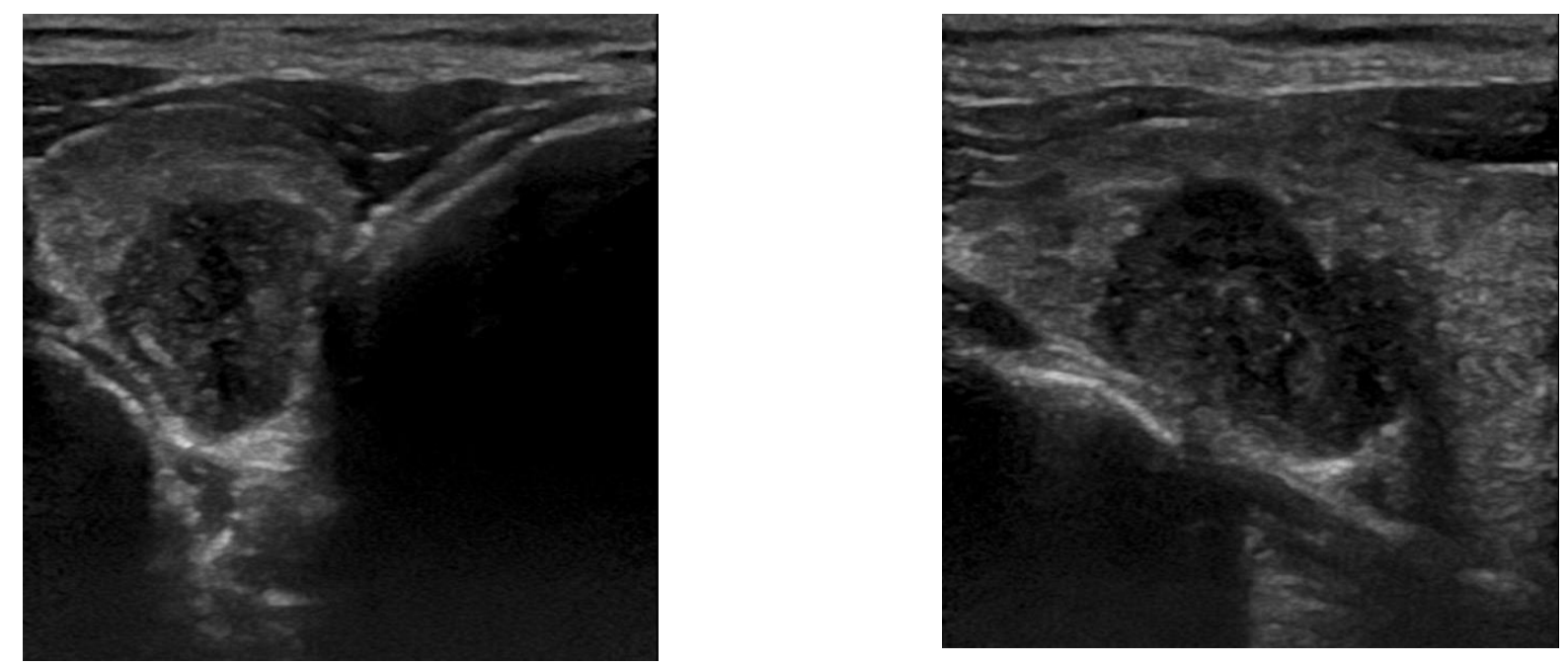

Case (6)

Female patient aged 54 years with right thyroid swelling.

\section{Ultrasound features of the nodule:}

- Partially cystic partially solid nodule is seen at the right thyroid lobe. The solid part is isoechoic with irregular margins and punctate echogenic foci. It measures about 12 x 13 $\mathrm{x} 15 \mathrm{~mm}$.

TIRADS level: TIRADS (5).

Pathology: Medullary thyroid carcinoma 


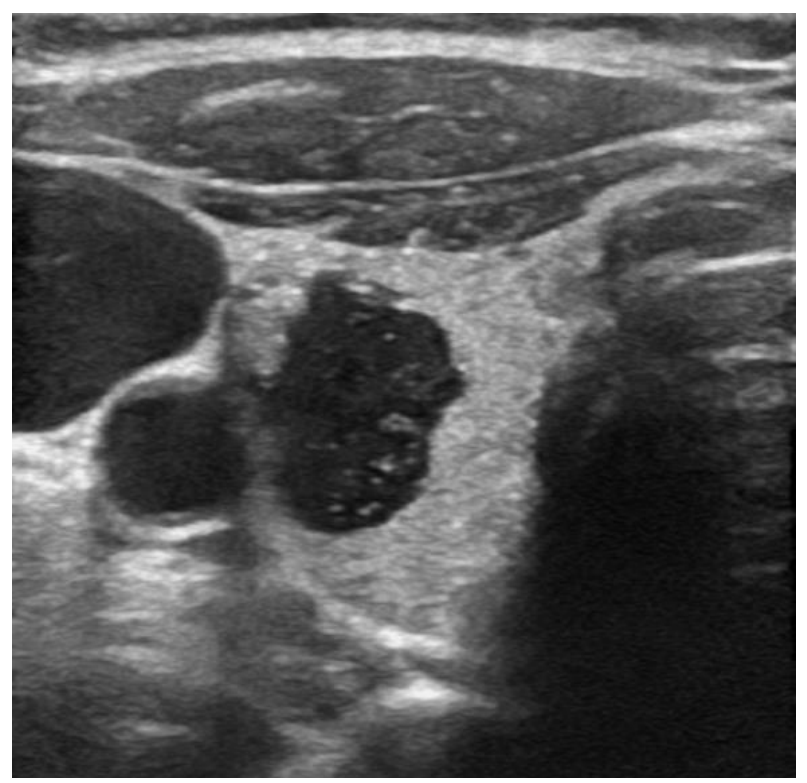

Discussion:

The differentiation of benign and malignant thyroid nodules is of great clinical significance. At present, although highresolution ultrasound remains the preferred method for evaluating thyroid nodules, the pathology of thyroid nodules is complex, the ultrasound findings of benign and malignant nodules often overlap, and the diagnostic results are closely related to the clinical experience of the ultrasound specialists. Due to the differences in the outcomes between hospitals and doctors, it is vital to establish a unified standard for the classification and evaluation of the severity of thyroid nodules, so that each physician can perform an objective and consistent evaluation of the nature (benign and malignant) of thyroid nodules (6).

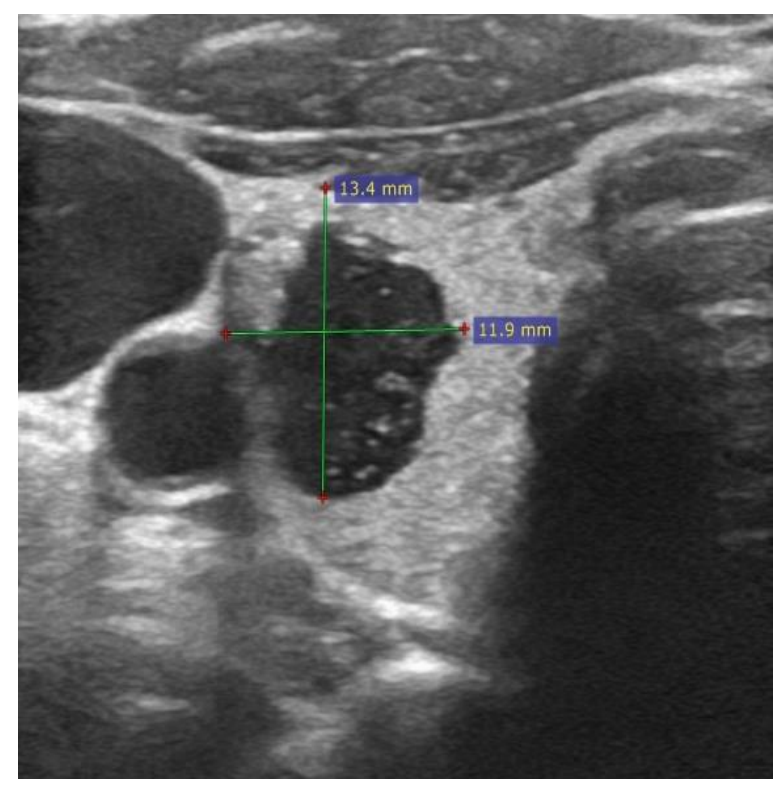

This study was conducted at BENHA University Hospitals aiming to assess the reliability of TIRADS in evaluation of thyroid nodules and its role in predicting thyroid malignancy.

Our study included a total of 30 cases diagnosed with thyroid lesions. Their mean age was 47 years (range, $21-71$ ). The study included 24 females $(80 \%)$, as well as 6 males (20\%). Comparing to (study 7): in which total of 602 cases with thyroid lesions were included. The sex-ratio was 0.36 (M/F). The mean age of patients was $50.9 \pm$ 14.8 years old (7).

In the current study, age was not a significant variable between benign and malignant cases $(p=0.76)$. The mean age 
for both groups was 47.39 and 45.71 years respectively. Similar to our results, (study 8) reported no significant difference between benign and malignant cases regarding age of the participants $(\mathrm{p}=0.347)$. The mean age of both groups was 58 and 50 years respectively.

In the current study, there was a significant difference between benign and malignant cases regarding TIRADS classification ( $\mathrm{p}<$ 0.001). Benign lesion was detected from TIRADS 1 to 4 (34.8\% were TIRADS $2 \&$ 3, 26.1\% was TIRADS 4 and $4.3 \%$ were TIRADS 1). Malignant lesions were distributed among TIRADS 4 \& 5 (85.7\% were TIRADS 5 and $14.3 \%$ ere TIRADS 4). It was evident that TIRADS classification of thyroid lesion had accuracy of $80 \%$ in detecting malignant lesions with $100 \%$ sensitivity and lower specificity $73.9 \%$.

Similar results to ours, were reported by another study, as there was a significant difference between benign and malignant cases regarding TIRADS findings $(\mathrm{p}=$ 0.003). There was a positive correlation between TI-RADS score and risk of malignancy (8).

In our study, lesion consistency was not significantly different between the benign and malignant cases $(p=0.57)$. Solid lesions were diagnosed in 52.2 and $71.4 \%$ of case in benign and malignant groups respectively.

The same study confirmed our findings, as lesion consistency by ultrasound did not differ between benign and malignant cases $(\mathrm{p}=0.545)(8)$.

The study conducted on 1989 (9) showed that solid echo-texture had sensitivity of $54.5 \%$ and specificity of $64.3 \%$. While in another study performed 2001, it got sensitivity of $83.4 \%$ and specificity of $81.8 \%$ of solid echo-texture of malignant nodules. $(9,10)$.

In the current study, the echogenicity of the detected lesions did not differ significantly between benign and malignant cases $(\mathrm{p}=$ 0.47). However, hypoechogenicity was present in 8.7 and $28.6 \%$ of benign and malignant cases respectively. It was previously reported that they could predict malignancy in thyroid nodules using hypoechogenicity as criteria with sensitivity of $87.1 \%$, specificity of $43.4 \%$ and positive predictive value of $11.4 \%$ (11).

In our study, lesion borders were significantly different between the two groups $(\mathrm{p}<0.001)$. Irregular borders were present in $57.1 \%$ of malignant cases, whereas it was not detected in benign cases. 
On the contrary, well defined margins were present in $91.3 \%$ of benign cases, while it was absent in malignant cases $(0 \%)$. In the same previous study it was reported that irregular margins could be considered as independent risk factor of malignancy with sensitivity of $77.5 \%$, specificity of $85 \%$ and positive predictive value of $30 \%$ (11).

In our study, calcifications were detected in 17.4 and $14.3 \%$ of benign and malignant cases respectively. However, there was a significant difference between both groups regarding the nature of echogenic foci $(\mathrm{p}<$ 0.001). In study conducted on 20013, sensitivity of microcalcification as a predictor of malignancy was $29 \%$ (11).

In the current study, all benign cases had wider orientation $(100 \%)$, while malignant cases had wider and taller orientations in 57.1 and $42.9 \%$ of cases respectively. Lesion orientation was significantly different between both groups $(p=0.009)$. In other study, taller than wide for predicting malignancy had sensitivity of $4.35 \%$, specificity of $100 \%$, positive predictive value of $100 \%$ and negative predictive value of $94.87 \%$ (12).

\section{Conclusion:}

In conclusion, the sonographic findings of thyroid nodules are different for benign and malignant lesions. We conclude that sonography can be helpful in categorization of a nodule in one of TIRADS categories. With the high sensitivity of TIRADS classification, minimal invasive diagnostic methods such as fine needle aspiration biopsy should be performed on thyroid nodules classified TIRADS $4 \& 5$.

TIRADS classification is reliable in predicting thyroid malignancy. It improves patient management and cost effectiveness.

\section{References}

1-Horvath E, Silva C, Majlis S, Rodriguez I, Skoknic V, Castro A, et al. (2017). Prospective validation of the ultrasound based TIRADS (Thyroid Imaging Reporting and Data System) classification: results in surgically resected thyroid nodules. European radiology, 27(6), 2619-2628.

2-Remonti L R, Kramer C K, Leitao C B, Pinto L C F, \& Gross J L. (2015). Thyroid ultrasound features and risk of carcinoma: a systematic review and metaanalysis of observational studies. Thyroid, 25(5), 538-550.

3-Angell, T. E., Maurer, R., Wang, Z., Kim, M. I., Alexander, C. A., Barletta, J. A, et al. (2019). A cohort analysis of clinical and ultrasound variables predicting cancer risk in 20,001 consecutive thyroid 
nodules. The Journal of Clinical Endocrinology \& Metabolism, 104(11), 5665-5672.

4-Russ, G., Bonnema, S. J., Erdogan, M. F., Durante, C., Ngu, R., \& Leenhardt, L. (2017). European Thyroid Association guidelines for ultrasound malignancy risk stratification of thyroid nodules in adults: the EU-TIRADS. European Thyroid Journal, 6(5), 225-237.

5-CARNES, N. 2018. Predicting risk of malignancy in patients with indeterminate thyroid nodules. Boston University.

6-Tan, H., Li, Z., Li, N., Qian, J., Fan, F., Zhong, H. et al. (2019). Thyroid imaging reporting and data system combined with Bethesda classification in qualitative thyroid nodule diagnosis. Medicine, 98(50).

7-Chaigneau, E., Russ, G., Royer, B., Bigorgne, C., Bienvenu-Perrard, M., Rouxel, et al. (2018). TIRADS score is of limited clinical value for risk stratification of indeterminate cytological results. European journal of endocrinology, 179(1), 13-20.

8-Ulisse, S., Bosco, D., Nardi, F., Nesca, A., D'Armiento, E., Guglielmino, V, et al. (2017). Thyroid imaging reporting and data system score combined with the new italian classification for thyroid cytology improves the clinical management of indeterminate nodules. International Journal of Endocrinology, 2017.
9-Aggarwal, S ; Jayaram, G ; Kakar, A ; Goel, G ; Prakash, R and Pant, C. (1989). Fine needle aspiration cytologic diagnosis of the solitary cold thyroid nodule. Comparison with ultrasonography, radionuclide perfusion study and xeroradiography. Acta cytologica, 33(1), 41-47.

10- Koike, E., Noguchi, S., Yamashita, H., Murakami, T., Ohshima, A., Kawamoto, H., et al. (2001). Ultrasonographic characteristics of thyroid nodules: prediction of malignancy. Archives of surgery, 136(3), 334-337.

11- Papini, E., Guglielmi, R., Bianchini, A., Crescenzi, A., Taccogna, S., Nardi, F., et al. (2002). Risk of malignancy in nonpalpable thyroid nodules: predictive value of ultrasound and color-Doppler features. The Journal of Clinical Endocrinology \& Metabolism, 87(5), 1941-1946.

12- Moifo, B ; Takoeta, E O ; Tambe, J ; Blanc, F and Fotsin, J G. (2013). Reliability of thyroid imaging reporting and data system (TIRADS) classification in differentiating benign from malignant thyroid nodules. Open J Radiol, 3(3), 103107.

To cite this article: Medhat M. Reffat, Maha Z. Bilal, Mona N. Elbeshbishi, Mai M. Elrakhawy. Validation of TIRADS in Evaluation of Thyroid Nodule. BMFJ 2021;38(2):613-642. DOI: $10.21608 / \mathrm{bmfj} .2020 .28840 .1253$ 